\title{
Safety assessment of Lactobacillus reuteri IDCC 3701 based on phenotypic and genomic analysis
}

\author{
Bo Som Lee ${ }^{1}$, O-Hyun Ban ${ }^{1,2}$, Won Yeong Bang ${ }^{1,2}$, Seung A Chae², Sangki Oh², Chanmi Park², Minjee Lee², \\ Soo-Jung Kim ${ }^{3}$, Jungwoo Yang ${ }^{2^{*}}$ and Young Hoon Jung ${ }^{1,4^{*}}$ (D)
}

\begin{abstract}
Purpose: Lactobacillus reuteri is one of the most largely studied human-commensal bacteria and widely used as a form of probiotics. Safety of probiotics has become increasingly important for human consumption due to increasing health-concerns in food industry. In this study, the safety of L. reuteri IDCC 3701 isolated from human breast milk was thoroughly investigated.

Methods: Whole-genome sequence analysis was performed to identify antibiotic resistance and toxigenicity of $L$. reuteri IDCC 3701. Phenotypic analysis such as minimal inhibitory concentration, $\beta$-hemolysis, extracellular enzyme activity, and the production of biological amines and L/D-lactate, was investigated. Finally, acute oral toxicity test was performed to access $L$. reuteri IDCC 3701 safety.

Results: Although multiple resistances to gentamicin and kanamycin were observed in $L$. reuteri IDCC 3701 , it was revealed that these resistances are intrinsic and not transferable through whole-genome analysis. In addition, various phenotypic analysis concerning hemolysis, enzyme activity, and D-lactate production did not show any negative results. Although L. reuteri IDCC 3701 harbors a histidine decarboxylase gene, no biogenic amines were detected. Finally, L. reuteri IDCC 3701 exhibited no evidence of acute toxicity according to an in vivo study.

Conclusion: Our findings demonstrate that L. reuteri IDCC 3701 is considered to be safe for human consumption as probiotics based on the in silico, in vitro and in vivo studies.
\end{abstract}

Keywords: Probiotics, Lactic acid bacteria, Lactobacillus reuteri, Safety evaluation

\section{Introduction}

Probiotics have recently begun to be considered as one of the most effective sources of functional foods (Byakika et al. 2019; Saarela 2019). Typically, Lactobacillus, Bifidobacterium, and Saccharomyces strains are representatives found in probiotic formulations for commercial markets (Ricci et al. 2017). Although these probiotics are prevalent in the intestine as beneficial microorganisms, they can

\footnotetext{
* Correspondence: yjw@ildong.com; younghoonjung@knu.ac.kr ${ }^{2}$ Ildong Bioscience, 17 Poseunggongdan-ro, Pyeongtaek-si, Gyeonggi-do 17957, Republic of Korea

'School of Food Science and Biotechnology, Kyungpook National University, Daegu 41566, Republic of Korea

Full list of author information is available at the end of the article
}

cause adverse events such as inflammatory bowel disease in rare case (Doron and Snydman 2015). Thus, the safety evaluation of probiotic strains has become the most fundamental criterion for human consumption in the food industry (Salvetti et al. 2016). Especially, the safety of new isolated species cannot be equal to that of pre-existing species, because each of probiotic strain has strain-specific characteristics (Alayande et al. 2020; Salvetti et al. 2016). Thus, individual strain should be evaluated for safety as probiotics independently.

Lactobacillus reuteri is an obligate heterofermentative species and has been widely reported to confer multiple beneficial effects by improving gut mucosal integrity and nutrient absorption (Hou et al. 2015; Mu et al. 2018;

(c) The Author(s). 2021 Open Access This article is licensed under a Creative Commons Attribution 4.0 International License, which permits use, sharing, adaptation, distribution and reproduction in any medium or format, as long as you give appropriate credit to the original author(s) and the source, provide a link to the Creative Commons licence, and indicate if changes were made. The images or other third party material in this article are included in the article's Creative Commons licence, unless indicated otherwise in a credit line to the material. If material is not included in the article's Creative Commons licence and your intended use is not permitted by statutory regulation or exceeds the permitted use, you will need to obtain permission directly from the copyright holder. To view a copy of this licence, visit http://creativecommons.org/licenses/by/4.0/ 
Zheng et al. 2020). Additionally, L. reuteri has been found in a variety of hosts, including human and animals (Duar et al. 2017), and also in various body parts, including the gastrointestinal tract and breast (Böttcher et al. 2008; Valeur et al. 2004), implying that L. reuteri could be one of the most prevalent and fundamental probiotic strains.

In this study, potential risks of $L$. reuteri IDCC 3701, isolated from breast milk, were assessed by genomic and phenotypic analysis as well as oral acute toxicity study. As genomic analysis, antibiotic resistance genes, virulence genes and mobile elements were identified. As phenotypic analysis, hemolytic activity, enzymatic activity, biogenic amines and L-/D-lactate production, and minimum inhibitory concentrations (MICs) were investigated. Thus, this study provides a comprehensive outline for safety assessment of $L$. reuteri strain.

\section{Materials and methods}

\section{L. reuteri IDCC 3701}

To isolate bacteria that producing lactate, diluted breast milk samples $(5 \mathrm{~mL} \times 3)$ were spread on MRS medium (BD Difco, Frankilin Lakes, NJ, USA) containing 0.5\% $(\mathrm{w} / \mathrm{v}) \mathrm{CaCO}_{3}$ in a static incubator (IST-4075R, SANYO, Osaka, Japan) with $0.5 \% \mathrm{CO}_{2}$ at $37{ }^{\circ} \mathrm{C}$. Then, we identified L. reuteri IDCC 3701 (ATCC BAA-2837) by 16s rRNA sequencing (GenBank no. KM453732) of a colony forming hollow. The strain was maintained MRS medium and stored in $20 \%(\mathrm{w} / \mathrm{w})$ glycerol at $-80{ }^{\circ} \mathrm{C}$ for further usage.

\section{Identification of antibiotic resistance, virulence genes, mobile elements, and biogenic amines relate genes}

The whole-genome sequencing of $L$. reuteri IDCC 3701 was performed using a PacBio RSII instrument with an Illumina platform (Macrogen, Seoul, Korea). A nucleotides-sequence was generated by single molecule real-time (SMRT) sequencing system. Contigs were constructed by pre-assembling seed reads, by generating a consensus sequence of the mapped reads, and by correcting and filtering the reads. A consensus sequence with higher quality was obtained after error-correction of the constructed contigs by Pilon (version 1.21).

Putative virulence factors were identified with the BLASTn algorithm using the virulence factor database (VFDB; version 2020.02.13; http://www.mgc.ac.cn/VFs/) with identity $>70 \%$, coverage $>70 \%$, and $E$ value $<1 \mathrm{E}-5$ (Chen et al. 2005). For putative antibiotic resistance genes, the assembled sequences were compared to the reference antibiotic resistance gene sequences in the ResFinder database (https://cge.cbs.dtu.dk/services/ ResFinder/) using ResFinder 3.2 software. The search parameters for the analysis were sequence identity $>80 \%$ and coverage $>60 \%$ (Zankari et al. 2012). Prophage regions were identified using PHASTER web-based program (Arndt et al. 2016). Transposases and conjugal transfer proteins were annotated using the BLASTP against on transposases and conjugal transfer proteins retrieved from GenBank. The genomic islands were predicted using the GIPSy program (Soares et al. 2016).

It has been reported that histidine decarboxylase, tyrosine decarboxylase, lysine decarboxylase, ornithine decarboxylase, phenylalanine decarboxylase, and the enzymes involved in agmatine deiminase pathway such as $\mathrm{N}$-carbamoylputrescine amidase and agmatinase were the key enzymes for biosynthesis of biogenic amines (Gardini et al. 2016). After building profile hidden Markov models (HMMs) representing the conserved amino acid sequence patterns in these enzymes, the candidate biogenic amine biosynthesis genes on the IDCC 3701 genome were searched using the hmmsearch tool in HMMER package (Eddy 2011) with the constructed profile HMMs. The candidate genes were confirmed using BLASTP tool in NCBI BLAST + and hmmscan tool in HMMER package against SWISS-PROT database and PFAM database, respectively.

\section{Determination of minimal inhibitory concentrations (MICs)}

L. reuteri IDCC 3701 was assessed for susceptibility to a variety of antibiotics including ampicillin, vancomycin, gentamicin, kanamycin, streptomycin, erythromycin, clindamycin, tetracycline, and chloramphenicol (SigmaAldrich, St. Louis, MO, USA), which are typically used to treat enterococcal infections (Wiegand et al. 2008). The plate was then incubated at $37{ }^{\circ} \mathrm{C}$ anaerobically for $18 \mathrm{~h}$ and the optical density was observed using a microplate reader (BioTek, Winooski, VT, USA).

\section{$\beta$-Hemolytic and enzymatic activities}

Overnight culture of L. reuteri IDCC 3701 was streaked on Sheep blood agar plates (BD Difco) and incubated at $37^{\circ} \mathrm{C}$ overnight. Then, the hemolytic potential of L. reuteri IDCC 3701 was evaluated by observing the cleared zones around the colonies. Staphylococcus aureus ATCC 25923 which was incubated in blood-based brain heart infusion (BHI) medium at $37{ }^{\circ} \mathrm{C}$ was used as a positive control for $\beta$-hemolysis by culturing on sheep blood agar plates.

Enzymatic activities were investigated using the API ZYM Kit (BioMérieux, Marcy-l'Etoile, France). In brief, $1.8 \times 10^{9} \mathrm{CFU} / \mathrm{mL}$ of $L$. reuteri $\mathrm{IDCC} 3701$ was incubated in MRS medium at $37^{\circ} \mathrm{C}$ for $4 \mathrm{~h}$ prior to loading into the API ZYM strips. Then, one drop of each of ZYM-A and ZYM-B reagents were added to each well. After $5 \mathrm{~min}$, color changes were observed and compared to the color reaction chart. 


\section{Biogenic amines and D-/L-lactate production}

Biogenic amines were measured according to Priyadarshani and Rakshit 2011. Briefly, the supernatant from overnight cultured L. reuteri IDCC 3701 was collected after centrifugation at $2300 \times g$ and $4{ }^{\circ} \mathrm{C}$ for $30 \mathrm{~min}$ and filtered with a $0.22-\mu \mathrm{m}$-pore size membrane. Prior to the quantification of the biogenic amines (BAs), $0.75 \mathrm{~mL}$ of supernatant was mixed with the equivalent of $0.1 \mathrm{M} \mathrm{HCl}$ and filtered with a $0.45-\mu \mathrm{m}$ membrane to extract the BAs. Then, $1 \mathrm{~mL}$ of the extracted BAs was incubated in a water bath at $70{ }^{\circ} \mathrm{C}$ for $10 \mathrm{~min}$, followed by the addition of $200 \mu \mathrm{L}$ of saturated $\mathrm{NaHCO}_{3}, 20 \mu \mathrm{L}$ of $2 \mathrm{M}$ $\mathrm{NaOH}$, and $0.5 \mathrm{~mL}$ of dansyl chloride $(10 \mathrm{mg} / \mathrm{mL}$ acetone). The derivatized BAs were mixed with $200 \mu \mathrm{L}$ of proline $(100 \mathrm{mg} / \mathrm{mL} \mathrm{H} 2 \mathrm{O})$ and incubated in the dark at room temperature for $15 \mathrm{~min}$. Acetonitrile (HPLC grade; Sigma-Aldrich) was added to the mixture to a total volume of $5 \mathrm{~mL}$. The derivatized BAs were separated and quantified by HPLC (LC-NETII/ADC, Jasco, Macclesfield, UK) using an Athena C18 column (4.6 mm $\times 250$ $\mathrm{mm}$, ANPEL Laboratory Technologies, Shanghai, China) and a UV detector (UV-2075 plus, Jasco, Macclesfield, $\mathrm{UK})$ at $254 \mathrm{~nm}$. Aqueous acetonitrile solution (67:33 of $\mathrm{H}_{2} \mathrm{O}, \mathrm{v} / \mathrm{v}$ ) was used as a mobile phase at a constant flow rate of $0.8 \mathrm{~mL} / \mathrm{min}$. Tyramine, histamine, putrescine, 2phenethylamine, and cadaverine were used as standards.

For the analysis of lactate, the supernatant from overnight cultured L. reuteri IDCC 3701 was collected by centrifugation at $2300 \times g$ and $4{ }^{\circ} \mathrm{C}$ for $30 \mathrm{~min}$ and then filtered with a $0.22-\mu \mathrm{m}$-pore size membrane. The quantities of L- and D-lactate in the supernatants were measured using an assay kit (Megazyme, Bray, Ireland) according to the manufacturer's protocol.

\section{Acute oral toxicity in rats}

All the rats were bred in Korea Testing and Research Institute (KTR; Hwasun-gun, Jeollanam-do, Korea) facilities which was maintained in an ambient temperature of $20.6-23.4{ }^{\circ} \mathrm{C}$ with a relative humidity of $47.6-59.7 \%$ on an automatically controlled 12/12 light cycle (lights off at 20:00 h) using 150-300 Lux. Less than 3 rats were in $(310 \mathrm{~W} \times 50 \mathrm{D} \times 20 \mathrm{H}) \mathrm{mm}$ cage and rats had free access to food (Rodent Diet 20 5053; Labdiet, St. Louis, MO, USA) and sufficient water. All efforts were made to minimize animal suffering and distress and the number of animals required for the production of reliable scientific data. The animal experiments (acute oral toxicity (AOT) test) in this study were conducted by KTR (TGK-2020-000008) under Animal protection act (no. 14651) and Laboratory animal act (no. 15278) by Korea government. Among four groups of three female rats (two groups aged nine weeks and two groups aged 10 weeks), each group was orally treated with $10 \mathrm{~mL}$ of $L$. reuteri IDCC $3701\left(13.8 \times 10^{10} \mathrm{CFU} / \mathrm{g}\right)$ per $\mathrm{kg}$ body weight (BW) (i.e., $300 \mathrm{mg} / \mathrm{kg} \mathrm{BW}$ or $2000 \mathrm{mg} / \mathrm{kg} \mathrm{BW}$ ). After observation of the clinical signs of morbidity, mortality, and BW during the 14 days (i.e., at the end of experimentation), animals were euthanized via isoflurane anesthesia.

\section{Results and discussion}

Whole genome analysis and determination of MICs

The whole-genome sequence of $L$. reuteri IDCC 3701 indicated that this strain harbors only chromosomal DNA without plasmid and the total length of the genome was about 2.09 million base pairs with a GC content of $38.87 \%$ and 2,087 functional genes (CDS) (Supplementary Fig. 1, Table 1).

For in silico safety analysis, we analyzed potential antibiotic resistance genes virulence factors, and mobile elements of $L$. reuteri IDCC 3701 . On the basis of the BLASTn algorithm and the VFDB, no putative virulence gene was identified in $L$. reuteri IDCC 3701 . In addition, no genes related to antibiotic resistance associated with aminoglycosides, beta-lactams, colistin, fosfomycin, fusidic acid, macrolides, nitroimidazole, oxazolidinone, phenicols, quinolones, rifampicin, sulphonamides, tetracyclines, trimethoprim, or glycopeptides were identified in the genome of L. reuteri IDCC 3701 (Table 2). Furthermore, mobile elements, such as transposes (64 genes), genomic island (15 genes), and prophage (0 gene) in the genome were analyzed in order to predict the possibility of antibiotic resistance gene transfer. However, these genes were meaningless due to absence of potential antibiotic resistance gene. Next, MICs of $L$. reuteri IDCC 3701 against nine antibiotics were analyzed to verify the safety of this strain. In results, L. reuteri IDCC 3701 was susceptible to all of the antibiotics, except for gentamicin and kanamycin (Table 2). The observed MICs for gentamicin and kanamycin were 2-fold and 4fold dilution above the EFSA cut-off value, respectively.

In this study, L. reuteri IDCC 3701 exhibited to be susceptible to all of the antibiotics, except for gentamicin and kanamycin (Table 2). Thus, the resistance against gentamicin and kanamycin seems to be intrinsic traits of this strain. Indeed, many Lactobacillus species have been found to be relatively tolerant to aminoglycoside antibiotics, such as gentamicin, kanamycin, and streptomycin

Table 1 Genome information of L. reuteri IDCC 3701

\begin{tabular}{ll}
\hline Items & Results \\
\hline Identification & Lactobacillus reuteri \\
ANI value (\%) & 99.997 \\
No. of 16s rRNA & 18 \\
Genome size (bp) & $2,085,171$ \\
GC contents (\%) & 38.87 \\
CDS & 2,087 \\
\hline
\end{tabular}


Table 2 Minimal inhibitory concentrations and antibiotic resistance gene of L. reuteri IDCC 3701

\begin{tabular}{|c|c|c|c|c|c|c|c|c|c|}
\hline Antibiotic $^{a}$ & AMP & VAN & GEN & KAN & STR & ERY & CLI & TET & $\mathrm{CHL}$ \\
\hline Cut-off value ${ }^{\mathrm{b}}(\mu \mathrm{g} / \mathrm{mL})$ & 2 & $n \cdot r^{c}$ & 8 & 64 & 64 & 1 & 4 & 32 & 4 \\
\hline Observed MIC & 0.5 & 512 & 16 & 256 & $32-64$ & $0.125-0.5$ & $0.125-0.5$ & 4 & 4 \\
\hline Assessment & $S^{d}$ & n.r & $\mathrm{R}^{\mathrm{e}}$ & $\mathrm{R}$ & S & S & S & S & $S$ \\
\hline Antibiotic resistance gene & n.d $d^{f}$ & n.d & n.d & n.d & n.d & n.d & n.d & n.d & n.d \\
\hline
\end{tabular}

${ }^{a} A M P$ ampicillin, VAN vancomycin, GEN gentamicin, KAN kanamycin, STR streptomycin, ERY erythromycin, CLI clindamycin, TET tetracycline, CHL chloramphenicol

${ }^{\mathrm{b}}$ EFSA (European Food Safety Authority) Guidelines, 2018. EFSA Journal, 16(3), 5206

cn.r not required

${ }^{d} S$ susceptible

${ }^{\mathrm{e}} R$ resistant

f $n . d$ not detected

(Danielsen and Wind 2003). This intrinsic resistance mechanism species might be due to membrane properties of these bacteria (Campedelli et al. 2018). In conclusion, L. reuteri IDCC 3701 has demonstrated to be safety with regard to antibiotic resistance.

\section{$\beta$-Hemolytic and enzymatic activities}

$\beta$-Hemolytic activity, which is a representative virulence factor enrolling in pathogenic invasion process to a host, should be absent in a probiotic strain to ensure safety. In this study, L. reuteri IDCC 3701 showed no hemolytic activity ( $\gamma$-hemolytic, no clear zone), while $S$. aureus ATCC 25923, a positive control, clearly showed $\beta$ hemolytic activity (a clear zone) on blood-based BHI agar (Supplementary Fig. 2). Enzyme activities involved in carbohydrate metabolism ( $\alpha$-galactosidase, $\beta$ galactosidase, and $\alpha$-galactosidase), lipid metabolism (esterase), and vitamin metabolism (acid phosphatase) were detected in L. reuteri IDCC 3701 (Table 3). Although $\beta$ glucosidase hydrolyzes glucose conjugates to generate beneficial secondary metabolites in the colon, it was also reported to produce potential carcinogenic compounds in rare cases (Cole and Fuller 1987). Meanwhile, $\beta$ glucuronidase which might directly link to carcinogenic compounds and thereby increase risk for colorectal cancer (Kim and Jin 2001), were not detected in L. reuteri IDCC 3701.

\section{Biogenic amines and lactate production}

Biogenic amines (BAs) production has been reported in some lactic acid bacteria (Beneduce et al. 2010; Özogul and Özogul, 2019; Spano et al. 2010). Large quantity of BAs is regarded as a potential health risk factor due to their toxicological effects (Spano et al. 2010). Histamine and tyramine are responsible for food-induced migraine, hypertensive crisis, and scombroid food poisoning (histamine intoxication) (Özogul and Özogul, 2019). Thus, profile search and homology search were performed using HMMER and BLAST algorithms. The genome harbors one gene (IDCC3701_1_01385) encoding a histidine decarboxylase. The gene has the high similarity with the reference sequence $(86.8 \%$ of $\mathrm{P} 00862$ from
Lactobacillus sp. 30A (GenBank Acc. No. AAB59151)) that was experimentally confirmed (Vanderslice et al. 1986). However, it was confirmed that other biosynthesis enzymes containing the tyrosine decarboxylase were not presented in this genome. Furthermore, in the supernatant of $L$. reuteri IDCC 3701, no biogenic amines such as tyramine, histamine putrescine, cadaverine, and 2-phenethylamine were detected in this strain (data not shown).

Some lactic acid bacteria can produce a mixture of L- and D-lactate (Stiles and Holzapfel 1997). Although D-lactate is not a highly toxic compound, D-lactate accumulation in blood (i.e., $3>\mathrm{mmol} / \mathrm{L}$, Petersen, 2005) might cause health problems due to

Table 3 Enzymatic activities of L. reuteri IDCC 3701

\begin{tabular}{|c|c|}
\hline Enzyme & L. reuteri \\
\hline Alkaline phosphate & $-{ }^{a}$ \\
\hline Esterase & $++^{b}$ \\
\hline Esterase lipase & - \\
\hline Lipase & - \\
\hline Leucine arylamidase & $++^{\mathrm{C}}$ \\
\hline Valine arylamidase & - \\
\hline Cystine arylamidase & - \\
\hline Trypsin & - \\
\hline a-chymotrypsin & - \\
\hline Acid phosphatase & ++ \\
\hline Naphthol-AS-BI-phosphohydrolase & ++ \\
\hline a-galactosidase & $+++^{d}$ \\
\hline$\beta$-galactosidase & +++ \\
\hline$\beta$-glucuronidase & - \\
\hline a-glucosidase & + \\
\hline$\beta$-glucosidase & - \\
\hline N-acetyl- $\beta$-glucosaminidase & - \\
\hline a-mannosidase & - \\
\hline a-fucosidase & - \\
\hline
\end{tabular}


Table 4 Body-weight changes of the rats administered L. reuteri IDCC 3701

\begin{tabular}{lllllll}
\hline Group & $\begin{array}{l}\text { Dose } \\
(\mathbf{g} / \\
\mathbf{k g ~ B W}\end{array}$ & \multicolumn{6}{l}{ Day after administration } \\
\cline { 3 - 7 } & $\mathbf{0}$ & $\mathbf{1}$ & $\mathbf{3}$ & $\mathbf{7}$ & $\mathbf{1 4}$ \\
\hline 9-week- & 300 & $193.0 \pm$ & $220.1 \pm$ & $225.0 \pm$ & $239.8 \pm$ & $254.9 \pm$ \\
aged & & 2.2 & 6.0 & 6.4 & 13.0 & 15.0 \\
& 2000 & $212.5 \pm$ & $231.5 \pm$ & $239.5 \pm$ & $250.1 \pm$ & $255.7 \pm$ \\
& & 10.6 & 14.8 & 16.5 & 14.3 & 18.6 \\
10-week- & 300 & $220.1 \pm$ & $220.9 \pm$ & $237.6 \pm$ & $257.5 \pm$ & $273.5 \pm$ \\
aged & & 7.9 & 6.4 & 9.9 & 4.0 & 3.3 \\
& 2000 & $217.3 \pm$ & $242.7 \pm$ & $249.5 \pm$ & $245.4 \pm$ & $251.5 \pm$ \\
& & 12.8 & 15.9 & 12.0 & 17.5 & 21.6 \\
\hline
\end{tabular}

${ }^{\mathrm{a}} B W$ body weight

a poor capacity of metabolizing D-lactate in human (Pohanka 2020; Puwanant et al. 2005). However, more recent studies have shown that D-lactate accumulation may only occur in cases of impaired Dlactate metabolism or a disturbed gastrointestinal function following any clinical symptoms, including short bowel syndrome (Connolly et al. 2005; Ku et al. 2006). In this study, L. reuteri IDCC 3701 was found to predominantly produces L-lactate (20.5 $\mathrm{mg} / \mathrm{mL}$, which is equivalent to $78.8 \%$ ) rather than D-lactate $(5.5 \mathrm{mg} / \mathrm{mL}, 21.2 \%)$, respectively.

\section{Single-dose acute oral toxicity study}

The in vivo toxicity of $L$. reuteri IDCC 3701 was investigated by a single-dose acute oral toxicity test. The results of the 14-day feeding to rats revealed that an oral administration of $L$. reuteri IDCC 3701 using $1.4 \times 10^{10}$ $\mathrm{CFU} / \mathrm{g}$ caused no abnormal findings in rats. In addition, there was no difference in gain-weight or loss of feed intake (Table 4), and also there was no change in appearance, behavior, or survival rate during the 14-day observation. At necropsy, no lesions due to the feeding of $L$. reuteri IDCC 3701 were detected in any organs. In sum, consumption of $0.3 \mathrm{~g}$ and $2 \mathrm{~g}$ of $L$. reuteri IDCC 3701 per $\mathrm{kg} \mathrm{BW}$ of rat was not nontoxic to the tested rats.

\section{Conclusion}

The safety of $L$. reuteri IDCC 3701 was demonstrated by genomic and phenotypic analyses and acute toxicity studies. The strain was found to be negative for antibiotic resistance and toxigenicity genes in the genome analysis. The strain did not produce any toxic compounds according to the phenotypic analysis. Furthermore, oral administration of this probiotic strain to rats showed no hazardous phenomena. Therefore, L. reuteri IDCC 3701 isolated from breast milk can be considered safe for human consumption as a probiotic.

\section{Supplementary Information}

The online version contains supplementary material available at https://doi. org/10.1186/s13213-021-01622-y

Additional file 1: Figure S1. Circular genome map and function genes of $L$. reuteri IDCC 3701. Figure S2. No $\beta$-hemolytic activity of $L$. reuteri IDCC 3701.

\section{Abbreviations}

AMP: Ampicillin; BA: Biogenic amine; BHI: Brain heart infusion;

CHL: Chloramphenicol; CLI: Clindamycin; ERY: Erythromycin; GEN: Gentamicin; HMM: Hidden Markov model; KAN: Kanamycin; MIC: Minimum inhibitory concentration; STR: Streptomycin; TET: Tetracycline; VAN: Vancomycin; VFDB: Virulence factor data base

\section{Acknowledgements}

This work was performed at the Research Center for Ildong Bioscience and at the School of Food Science and Biotechnology in Kyungpook National University.

\section{Authors' contributions}

Bo Som Lee and O-Hyun Ban performed experiments and analyzed the data. Won Yeong Bang, Seung A Choi, and Sangki Oh helped to perform experiments and analyze the data. Minjee Lee and Chanmi Park helped to finalize the manuscript. Jungwoo Yang and Young Hoon Jung designed and wrote the manuscript. Young Hoon Jung supervised the project. Soo Jung Kim aided in interpreting the results and provided critical revision. All authors read and approved the final manuscript.

\section{Funding}

This work was supported by Ildong Bioscience and also supported by the National Research Foundation of Korea (NRF) grant funded by Korea government (Ministry of Science and ICT, MSIT; No. 2020R1C1C1005251).

Availability of data and materials

All data generated or analyzed during this study are included in this published article, while 16S rDNA sequences of L. reuteri IDCC 3701 are deposited to NCBI Genome Information and available under the accession number, KM453732 (https://www.ncbi.nlm.nih.gov/nuccore/719239383/).

Ethics approval and consent to participate

Not applicable

Consent for publication

All authors read and approved the final manuscript for publication.

\section{Competing interests}

The authors declare that they have no competing interests.

\section{Author details}

${ }^{1}$ School of Food Science and Biotechnology, Kyungpook National University, Daegu 41566, Republic of Korea. ${ }^{2}$ ldong Bioscience, 17 Poseunggongdan-ro, Pyeongtaek-si, Gyeonggi-do 17957, Republic of Korea. ${ }^{3}$ Department of Integrative Food Bioscience and Biotechnology, Chonnam National University, Gwangju 61186, Republic of Korea. ${ }^{4}$ Institute of Fermentation Biotechnology, Kyungpook National University, Daegu 41566, Republic of Korea.

Received: 19 November 2020 Accepted: 26 January 2021

Published online: 15 February 2021

\section{References}

Alayande KA, Aiyegoro OA, Nengwekhulu TM, Katata-Seru L, Ateba CN (2020) Integrated genome-based probiotic relevance and safety evaluation of Lactobacillus reuteri PNW1. PLoS One 15:e0235873

Arndt D, Grant J, Marcu A, Sajed T, Pon A, Liang Y et al (2016) PHASTER: a better, faster version of the PHAST phage search tool. Nucleic Acids Res 44:W16-W21

Beneduce L, Romano A, Capozzi V, Lucas P, Barnavon L, Bach B et al (2010) Biogenic amine in wines. Ann Microbiol 60:573-578 
Böttcher MF, Abrahamsson TR, Fredriksson M, Jakobsson T, Björkstén B (2008) Low breast milk TGF-beta2 is induced by Lactobacillus reuteri supplementation and associates with reduced risk of sensitization during infancy. Pediatr Allergy Immunol 19:497-504

Byakika S, Mukisa IM, Byaruhanga YB, Muyanja C (2019) A review of criteria and methods for evaluating the probiotic potential of microorganisms. Food Rev Int 35:427-466

Campedelli I, Mathur H, Salvetti E, Clarke S, Rea MC, Torriani S et al (2018) Genuswide assessment of antibiotic resistance in Lactobacillus spp. Appl Environ Microbiol 85:e01738-e01718

Chen L, Yang J, Yu J, Yao Z, Sun L, Shen Y et al (2005) VFDB: a reference database for bacterial virulence factors. Nucleic Acids Res 33:D325-D328

Cole C, Fuller R (1987) The effect of dietary fat and yoghurt on colonic bacterial enzymes ( $\beta$-glucosidase and $\beta$-glucuronidase) associated with colon cancer. Food Microbiol 4:77-81

Connolly E, Abrahamsson T, Björkstén B (2005) Safety of D(-)-lactic acid producing bacteria in the human infant. J Pediatr Gastroenterol Nutr 41:489-492

Danielsen M, Wind A (2003) Susceptibility of Lactobacillus spp. to antimicrobial agents. Int J Food Microbiol 82:1-11

Doron S, Snydman DR (2015) Risk and safety of probiotics. Clin Infect Dis 60: S129-S134

Duar RM, Frese SA, Lin XB, Fernando SC, Burkey TE, Tasseva G et al (2017) Experimental evaluation of host adaptation of Lactobacillus reuteri to different vertebrate species. Appl Environ Microbiol 83:e00132-e00117

Eddy SR (2011) Accelerated profile HMM searches. PLoS Comput Biol 7(10): e1002195

Gardini F, Özogul Y, Suzzi G, Tabanelli G, Özogul F (2016) Technological factors affecting biogenic amine content in foods: a review. Front Micorbiol 7:1218

Hou C, Zeng X, Yang F, Liu H, Qiao SJ (2015) Study and use of the probiotic Lactobacillus reuteri in pigs: a review. Anim Sci Biotechnol 6:14

Kim DH, Jin YH (2001) Intestinal bacterial $\beta$-glucuronidase activity of patients with colon cancer. Arch Pharm Res 24:564-567

Ku WH, Lau DCY, Huen KF (2006) Probiotics provoked D-lactic acidosis in short bowel syndrome: case report and literature review. HK J Paediatr 11:246-254

Mu Q, Tavella VJ, Luo XM (2018) Role of Lactobacillus reuteri in human health and diseases. Front Microbiol 9:757

Özogul T, Özogul F (2019) Chapter 1: biogenic amines formation, toxicity, regulations in food. In: Saad B, Tofalo $R$ (eds) Biogenic amines in food: Analysis, occurrence and toxicity. Royal Society of Chemistry, London, pp 1-17

Petersen C (2005) D-Lactic acidosis. Nutr Clin Pract 20:634-645

Pohanka M (2020) D-Lactic acid as a metabolite: toxicology, diagnosis, and detection. Biomed Res Int 3419034:9

Priyadarshani WM, Rakshit SK (2011) Screening selected strains of probiotic lactic acid bacteria for their ability to produce biogenic amines (histamine and tyramine). Int J Food Sci Technol 46:2062-2069

Puwanant M, Mo-Suwan L, Patrapinyokul S (2005) Recurrent D-lactic acidosis in a child with short bowel syndrome. Asia Pac J Clin Nutr 14:195-198

Ricci A, Allende A, Bolton D, Chemaly M, Davies R, Girones R et al (2017) Update of the list of QPS-recommended biological agents intentionally added to food or feed as notified to EFSA 6: suitability of taxonomic units notified to EFSA until March 2017. EFSA J 15:e04663

Saarela MH (2019) Safety aspects of next generation probiotics. Curr Opin Sci 30: 8-13

Salvetti E, Orrù L, Capozzi V, Martina A, Lamontanara A, Keller D et al (2016) Integrate genome-based assessment of safety for probiotic strains: Bacillus coagulans GBI-30, 6068 as a case study. Appl Microbiol Biotechnol 100:45954605

Soares SC, Geyik H, Ramos RT, De Sá PH, Barbosa EG, Baumbach J et al (2016) GIPSy: genomic island prediction software. J Biotechnol 232:2-11

Spano G, Russo P, Lonvaud-Funel A, Lucas P, Alexandre H, Grandvalet C et al (2010) Biogenic amines in fermented foods. Eur J Clin Nutr 64: S95-S100

Stiles ME, Holzapfel WH (1997) Lactic acid bacteria of foods and their current taxonomy. Int J Food Microbiol 36:1-29

Valeur N, Engel P, Carbajal N, Connolly E, Ladefoged K (2004) Colonization and immunomodulation by Lactobacillus reuteri ATCC 55730 in the human gastrointestinal tract. Appl Environ Microbiol 70:1176-1181

Vanderslice P, Copeland WC, Robertus JD (1986) Cloning and nucleotide sequence of wild type and a mutant histidine decarboxylase from Lactobacillus 30a. J Biol Chem 261(32):15186-15191
Wiegand I, Hilpert K, Hancock REW (2008) Agar and broth dilution methods to determine the minimal inhibitory concentration (MIC) of antimicrobial substances. Nat Protoc 3:163-175

Zankari E, Hasman H, Cosentino S, Vestergaard M, Rasmussen S, Lund O et al (2012) Identification of acquired antimicrobial resistance genes. J Antimicrob Chemother 67:2640-2644

Zheng J, Wittouck S, Salvetti E, Franz CMAP, Harris HMB, Mattarelli P et al (2020) A taxonmonic note on the genus Lactobacillus: description of 23 novel genera, emended description of the genus Lactobacillus Beijerinck 1901, and union of Lactobacillaceae and Leuconostocaceae. Int J Syst Evol Microbiol 70: 2782-2858

\section{Publisher's Note}

Springer Nature remains neutral with regard to jurisdictional claims in published maps and institutional affiliations.

\section{Ready to submit your research? Choose BMC and benefit from:}

- fast, convenient online submission

- thorough peer review by experienced researchers in your field

- rapid publication on acceptance

- support for research data, including large and complex data types

- gold Open Access which fosters wider collaboration and increased citations

- maximum visibility for your research: over $100 \mathrm{M}$ website views per year

At BMC, research is always in progress.

Learn more biomedcentral.com/submissions 